\title{
Output, stock markets and macro-policy measures in a Keynesian portfolio model
}

\author{
Toichiro Asada*, Matthieu Charpe ${ }^{* *}$, Peter Flaschel***, \\ Christopher Malikane ****, Tarik Mouakil $^{* * * *}$ and \\ Christian R. Proaño******
}

\begin{abstract}
We present a simple macrodynamic model of the real-financial markets inter action with a dynamic multiplier representing the goods market and a structured portfolio choice between money holdings and equities. This is contrasted with Blanchard's (I98I) alternative approach, where interest-bearing bonds and equities are perfect substitutes and are subject to myopic perfect foresight, with the result that the usual saddle-point dynamics is established, and where therefore the jump-variable technique of the rational expectations approach is needed in order to tame the explosive nature of the model by assumption. We consider this latter representation a very virtual one in contrast to our descriptive treatment of the interaction of the real with the financial markets. Our implied dynamical system has three dimensions: output, share prices and capital gains expectations. We show that this model exhibits a financial accelerator mechanism that endangers the stability of its stationary solution, at least when it becomes sufficiently strong. Furthermore, we show that this type
\end{abstract}

* Department of Economics, Chuo University, Tokyo, Japan.

** International Labor Organisation, Genéve, Switzerland.

*** Department of Business Administration and Economics, Bielefeld University, Bielefeld, Germany.

**** School of Economic and Business Sciences, University of the Witwatersrand Johannesburg, South Africa.

***** Centre for Financial Analysis and Policy, University of Cambridge, Cambridge, United Kingdom.

****** The New School for Social Research, New York, and Macroeconomic Policy Institute (IMK), Düsseldorf, Germany.

Correspondence Address:

Peter Flaschel, Bielefeld Graduate School of Economics and Management, Bielefeld University, P.O. Box I00I3I, 3350r Bielefeld, Germany, e-mail: pflaschel@wiwi.uni-bielefeld.de.

Received I4 March 20IO, accepted 24 October 2010

(C) INTERVENTION 8 (2), 2OII, 34I-360 
of instability can definitely be overcome if actual capital gains are taxed to a sufficient degree. This tax policy is therefore effective as compared to an interest rate policy of Taylor type, showing that monetary policy may not impact the markets for risky financial assets, unless it is interpreted as state of confidence for the current situation of the economy and may therefore be fairly overrated in the current macrodynamic literature. By contrast, all policy measures that stabilise the profit rate of the economy may add to the stability implications of the assumed Asada et al. (2010) type tax on capital gains.

JEL classifications: EI2, E32

Keywords: portfolio choice, financial accelerators, fiscal policy, monetary policy

\section{Portfolio choice and real-financial market interactions}

As the actual global financial crisis makes clear in a quite unpleasant manner, market participants in the financial markets do not always behave rationally, as it is assumed in the great majority of macroeconomic models nowadays. This has far-reaching consequences for the conduct of economic policy. Indeed, in a world where agents are rational and the financial markets are complete, the best strategy for monetary policy to follow is the targeting of goods price inflation. In contrast, when financial markets are driven by non-rational expectations and are incomplete, an inflation targeting strategy may not be sufficient for the achievement of macroeconomic stabilisation.

In Asada et al. (2010) we have used the six dimensional K(eynes)M(etzler)G(oodwin) monetary growth model as the basis for an extension towards a Tobin (1982) treatment of the financial sector by way of a module representing portfolio choice. In this paper, by contrast, we drastically simplify the specification of the real part of the KMG economy, by ignoring inflation and growth altogether, and by representing the quantity adjustment process by a simple dynamic multiplier approach as in Blanchard (198I). This also removes the Metzlerian inventory accelerator mechanism as a source of instability besides the wage price spiral and makes the real part of the economy a stable one from its partial perspective.

Representative of traditional macroeconomics, Turnovsky (1995) makes use of a dynamic portfolio balance macroeconomic model where he considers the following representation of the financial part of his portfolio model. In order to make it comparable to our subsequent modelling of such an approach we however ignore here inflation and also transfer the Turnovsky model to the notation used in this paper (see for the notation the appendix to this article).

$$
\begin{aligned}
& M^{d}=f_{m}(Y, i, r)(M+B+p K), \\
& B^{d}=f_{b}(Y, i, r)(M+B+p K), \\
& p K=f_{k}(Y, i, r)(M+B+p K),
\end{aligned}
$$


with

$$
f_{m 2}(Y, i, r)+f_{b 2}(Y, i, r)+f_{k 2}(Y, i, r) \equiv 0, f_{m 3}(Y, i, r)+f_{b 3}(Y, i, r)+f_{k 3}(Y, i, r) \equiv 0,
$$

in particular (and $M^{d}+B^{d}+p K=M+B+p K$ ) and with the gross substitution assumption being made.

We concentrate on financial markets, treat output $Y$ as a parameter, and consider the three markets in equilibrium with the corresponding supplies. Due to Walras' law of stocks we need to consider only two of these equilibrium conditions and use them in order to determine the two endogenous variables $i$, the rate of interest and $r$, the rate of profit.

This is a very tranquil representation of financial markets where expectations play no role at all, since there are no capital gains, and where the rate of profit is determined through a resale market for physical capital, an assumption usually not made in Keynesian approaches to macrodynamics (see Sargent [1987, ch. III]). In this model monetary policy can influence the rate of profit of the economy solely through the financial market structure of the economy which is independent of the conflict over income distribution between capital and labour.

We view the above structure as not being adequate for building a Keynesian portfolio model of the real-financial market interaction, particularly when the current situation in financial markets is taken into account. As Keynes wrote already in I936:

"Speculators may do no harm as bubbles on a steady stream of enterprise. But the position is serious when enterprise becomes the bubble on a whirl-pool of speculation. When the capital development of a country becomes a by-product of the activities of a casino, the job is likely to be ill-done. The measure of success attained by Wall Street, regarded as an institution of which the proper social purpose is to direct new investment into the most profitable channels in terms of future yield, cannot be claimed as one of the outstanding triumphs of laissez-faire capitalism - which is not surprising, if I am right in thinking that the best brains of Wall Street have been in fact directed towards a different object." (Keynes 1936: 159)

It is in our view impossible to make sense out of this quotation on the basis of the above portfolio approach. As in Asada et al. (2OIO), we therefore assume here the following baseline modification of the asset demand functions of the Turnovsky approach, employing now equities $\bar{E}$, issued by firms in the past, and the share prices $p_{e}$ in place of the physical capital stock $K$. We assume for analytical simplicity that all profits are paid out as dividends and, on this basis, get for the portfolio demand structure the model:

$$
\begin{aligned}
& M^{d}=f_{m}\left(i, r_{e}^{e}\right) W_{c}^{n}, \\
& B^{d}=f_{b}\left(i, r_{e}^{e}\right) W_{c}^{n}, \\
& p_{e} E^{d}=f_{e}\left(i, r_{e}^{e}\right) W_{c}^{n},
\end{aligned}
$$

with the balance sheet reallocation condition:

$$
f_{m}\left(i, r_{e}^{e}\right)+f_{b}\left(i, r_{e}^{e}\right)+f_{e}\left(i, r_{e}^{e}\right) \equiv 1
$$


and the definition of exogenously given nominal value of private wealth:

$$
W_{c}^{n}=\bar{M}+\bar{B}+p_{e} \bar{E}
$$

based on the past issue of government bonds $\bar{B}$ and Central Bank money $\bar{M}$ besides the considered equity issue of firms.

For the expected rate of return on equities we moreover get

$$
r_{e}^{e}=\frac{p Y-w L^{d}}{p_{e} \bar{E}}+\bar{\pi}_{e}^{e}=r_{y} Y / q+\bar{\pi}_{e}^{e},
$$

by using Tobin's average $q=\frac{p_{e} \bar{E}}{p K}$

on the basis of the profit function $r=r_{v} Y$. The essential differences to the Turnovsky model are the variable $r / q$ in place of solely $r$, the expression $p_{e} E$ in place of $p K$ and most importantly the inclusion of capital gain expectations in the expected rate of return on equities $r / q+\pi_{e}^{e}$.

We have assumed a given amount of equities, exclude price level changes and capital accumulation, and normalise for reasons of notational simplicity the given ratio $\frac{\bar{E}}{p K}$ by 1 and thus can identify Tobin's $q$ with the share price $p_{e}$ The issue of new safe assets and new equities, and thus the accumulation of wealth is neglected in this dynamical analysis of the short run. It is however treated in detail in Asada et al. (20IO).

The variable $i$ represents the nominal interest rate, which is assumed to be fixed by the central bank at each moment in time according to the rule ( $p$ being constant):

$$
i=i(Y)=i_{o}+i_{y}\left(Y-Y_{o}\right), \quad i_{y}>0 .
$$

Note that even though the stock of the financial assets: safe money $M_{2}=M+B$, and equities $E$ is considered as exogenous, $M, B$ and of course $p_{e}$ are determined through the above portfolio equations, since the central bank has to adjust to the demands of households with respect to the two assets $M, B$ in the case of a given rate of interest, transforming the initially given values $\bar{M}_{2}=\bar{M}+\bar{B}$ into the components of $\bar{M}_{2}$ that are desired by the asset holders now. Our model is formulated below in a way such that the sum of money holdings remains fixed in time, unless the central bank is buying equities in place of fix-price bonds through its open market operations.

The asset holders for example increase their short-term bond holdings (saving deposits) in the case of an increase in the short term interest rate $i$ as in a Baumol cash-management inventory choice process, where we now neglect the influence of output of the Turnovsky approach. Changing interest rates therefore affect the cash management of asset holders, a fairly trivial and tranquil component within their whole portfolio choice, but do not affect the equity demand of asset holders, since they only consider their $M_{2}$ holdings (hoardings) as alternative to their equity holdings (in particular when the stock market is under pressure). Money holdings $M_{2}=M(i)+B(i)$ and equities $E$ are thus (imperfect) substitutes, but not the internal restructuring of $M_{2}$ as such, which only determines the transactions demand for money at each moment in time. 
In order to discuss the distinction to the Turnovsky model as succinctly as possible, we therefore simplify - following the above observations - the structure of the asset holders' portfolio choices further. In the equations that follow we have now added the assumptions that the decision between total money and equities has priority for asset holders and is done solely in view of the expected rate of return on equities as far as equity demand is concerned. Decisions on the stock market are therefore independent of the nominal rate of interest which is only relevant for asset holders' cash management within the money demand $M_{2}=M+B$, i.e. the money demand for $M_{2}$ does also not depend on the nominal rate of interest. This gives the following hierarchy of portfolio reallocation decisions within the balance sheet of asset holders:

$$
\begin{aligned}
& p_{e} \bar{E}=p_{e} E^{d}=f_{e}\left(r_{e}^{e}\right)\left(p_{e} \bar{E}+\bar{M}_{2}\right), \\
& \bar{M}_{2}=M_{2}^{d}=f_{b e}\left(r_{e}^{e}\right)\left(p_{e} \bar{E}+\bar{M}_{2}\right), \quad f_{e}(\cdot)+f_{b e}(\cdot)=1, \\
& B \quad=B^{d}=f_{b m}(i) \bar{M}_{2}, \quad f_{b m}(i)=1-f_{m}(i) \\
& M \quad=M^{d}=f_{m}(i) \bar{M}_{2}, \quad \bar{M}_{2}=\bar{M}+\bar{B} .
\end{aligned}
$$

Equity demand (vs. hoarding) represents therefore the crucial part in the decisions made in financial markets, while cash management between money $M=M_{1}$ and $B$ is a relatively trivial matter and for example executed by way of Baumol's well-known inventory approach, leading to the formula

$$
M^{d}=f_{m}\left(i, \bar{M}_{2}\right)=\sqrt{\frac{2 \phi\left(\bar{M}_{2}\right)}{i}}, \quad \text { i.e., } \quad i=\frac{2 \phi\left(\bar{M}_{2}\right)}{\bar{M}^{2}} .
$$

Asset holders determine transactions demand $M^{d}(i)$ as component of their total money holdings $\bar{M}_{2}$ which is available for portfolio choice at each moment in time. Before that they however consider their portfolio choice between $M_{2}$ and $E$ and do this under normal conditions with a strong focus on equity as the central component of their wealth, depending positively on the expected rate of return $r_{e}^{e}$ on such equities. They would demand more (less) equities than they are currently holding if this rate is higher (lower) than its steady state value. This determines the share price $p_{e}(=q)$ on the basis of a given rate of profit $r$ and given capital gains expectations $\pi_{e}^{e}$.

Note that asset holders must hold $\bar{E}, \bar{M}_{2}=\bar{M}+\bar{B}$ again at the end of the trading day, since standard open market policies of the central bank cannot change $\bar{M}_{2}$. The change in the stock price $p_{e}$ therefore just induces asset holders to hold their initial stocks again, implying that $M_{2}$ is a given magnitude in this setup. Note also that in times of stress on the equity market, where people want to go into money hoarding, the equity price will fall significantly without the possibility for asset holders as a whole to change their actual stock of equities.

In stock market equilibrium we have again $E^{d}=\bar{E}$ but due to trading process in the background of this situation, we have a new share price $p_{e}$ and thus during the process also 
a new money demand $M_{2}$. But at the end of it, the supply of equities is again held by asset holders and the demand for $M_{2}$ back at its given stock. We thus have an endogenous adjustment of $M(i)$ and $B(i)$, but not of $M_{2}$. The above portfolio structure implies that the central bank's monetary policy can only affect the asset markets through effects on the rate of profit of firms $r$ or the expectations of capital gains $\pi_{e}^{e}$.

It is easy to show that excess demand in asset markets is a negative function of the share price $p_{e}$. In disequilibrium the share price is therefore adjusting towards its equilibrium value, all other variables kept fixed for the moment. The equilibrium share price represents therefore an attractor of disequilibrium share prices. We assume that this ultra short-run process occurs in virtual time so that the market for equities is always cleared. Comparative static exercises then easily show that the equilibrium value of the stock price depends positively on the rate of profit and also positively on capital gains expectations, as one would have expected it to be true. We disregard changes in the stocks of capital and equities here and also changes in the price level $p$, since we assume zero inflation.

We however now make use of a further change in the description of the equity market, by making use of an adjustment rule for stock prices in place of an equilibrium condition. This can be formally justified by the argument that such a disequilibrium approach becomes unavoidable as soon as the capital account in open economies is considered in a continuous time model. Moreover, this is just another example of a stock adjustment principle from the literature which can be justified through the introduction of (some) adjustment costs.

$$
\hat{p}_{e}=\dot{p}_{e} / p_{e}=\beta_{e}\left[\alpha_{e} \frac{p_{e} E^{d}-p_{e} \bar{E}}{p_{e} \bar{E}}\right]=\beta_{e} \alpha_{e}\left[f_{e}\left(r / q+\pi_{e}^{e}\right)\left(1+\frac{M_{2}}{p_{e} \bar{E}}\right)-1\right]=\hat{q},
$$

where $\alpha_{e}$ describes the planned flows that are implied by the considered stock disequilibrium $\frac{p_{e} E^{d}-p_{e} \bar{E}}{p_{e} \bar{E}}$ and where $\beta_{e}$ determines the strength by which equity prices respond to these planned flows. Note that these flows are notational ones, since asset markets must settle at $\bar{E}$ when the trading day at , Wall-Street comes to an end. Together with the real disequilibria considered below we arrive in this paper for the first time - as compared to the portfolio equilibria considered in Asada et al. (20IO) and related work - at a Dynamic General Disequilibrium model which, when Stochastic terms are added, would provide a baseline DSGD approach in comparison to the DSGE approaches of the mainstream literature.

We couple this share price dynamic with the Blanchard (198I) type model (with adaptive capital gains expectations) where aggregate demand is dependent on Tobin's $q$ in its deviation from its steady state value $q_{o}{ }^{1}$ in place of the real rate of interest. This gives

$$
\begin{aligned}
& \dot{Y}=\beta_{y}\left(Y^{d}-Y\right)=\beta_{y}\left(\left(a_{y}-1\right) Y+a_{q}\left(q-q_{o}\right)+\bar{G}\right), \quad a_{y} \in(0,1), \\
& \dot{\pi}_{e}^{e}=\beta_{\pi}\left[\hat{q}-\pi_{e}^{e}\right]
\end{aligned}
$$


as output adjustment process and as capital gains expectations adjustment process. We get thereby the $3 \mathrm{D}$ dynamics in $Y, q, \pi_{e}^{e}$. In order to make these dynamics complete we assume that there holds:

$$
r=\frac{p Y-w L^{d}}{p K}=\frac{Y}{K}(1-\omega / z)=r_{y} Y, \quad \omega=w / p, \quad z=Y / L^{d},
$$

by assuming a constant value for the labour productivity coefficient $z>w / p$. This gives in sum:

$$
\begin{aligned}
\dot{Y} & =\beta_{y}\left(a_{y} Y+a_{q}\left(q-q_{o}\right)+\bar{G}-Y\right) \\
\hat{q} & =\beta_{e} \alpha_{e}\left[f_{e}\left(r_{y} Y / q+\pi_{e}^{e}\right)\left(1+\frac{M_{2}}{p_{e} \bar{E}}\right)-1\right] \\
\dot{\pi}_{e}^{e} & =\beta_{\pi}\left[\hat{q}-\pi_{e}^{e}\right]
\end{aligned}
$$

with the following Jacobian matrix

$$
J=\left(\begin{array}{lll}
- & + & 0 \\
+ & - & + \\
+ & - & \pm
\end{array}\right)
$$

at the steady state

$$
Y_{o}=\frac{\bar{G}}{1-a_{y}}, \quad \pi_{e}^{e}=0, \quad f_{e}\left(r_{y} Y_{o} / q_{o}\right)\left(1+\frac{M_{2}}{p_{e} \bar{E}}\right)=1 .
$$

It is standard to assume that the marginal propensity to spend is less than one and there holds of course $f_{e}^{\prime}>0$. And the equilibrium value of $q$ is easily shown to be uniquely determined (and positive if the function $f_{e}$ is chosen in an appropriate way).

\section{Stability by assumption versus stability results in models of the real-financial market interaction}

We now investigate the stability of the considered portfolio driven multiplier dynamics. We do this first for the neoclassical limit case of perfect asset substitution and perfect foresight, which however has nothing in common with the situations where deviations from these perfections occur.

\section{I The Neoclassical Limit Case (Blanchard 198I)}

Neoclassical theory often considers the case of perfect asset substitution coupled with myopic perfect foresight. In the present situation this can only be done when interest matters in the demand function for equities. After re-inserting the interest rate into the $f_{e}$ equation, in the 
form $r_{e}^{e}-i$, we then assume $\alpha_{e}=\infty$ in order to return to a stock equilibrium approach and then with respect to asset substitution in addition $f_{e}^{\prime}=\infty$ in order get the case of perfect asset substitution. Assuming finally $\beta_{\pi}=\infty$ gives rise to myopic perfect foresight and yields the following simple situation:

$$
i(Y)=r(Y)+\hat{q} \quad \text { or } \quad \dot{q}=i(Y) q-r(Y), \quad i \text { given. }
$$

There is thus now only a single law of motion for asset markets where the rate of change of Tobin's $q$ now depends positively on its level and where $Y$ is now negatively operating on this rate of change, just the opposite of the situation we had in the case of imperfect asset substitutability.

We only briefly describe how such dynamics, when integrated with output dynamics, works in this ideal neoclassical world of uniform rates of return. Thus, the following system has to be studied:

$$
\begin{aligned}
& \dot{Y}=\beta_{y}\left(a_{y} Y+a_{q}\left(q-q_{o}\right)+\bar{G}-Y\right), \\
& \dot{q}=i(Y) q-r_{y} Y, \quad i=i_{o}+i_{y}\left(Y-Y_{o}\right) .
\end{aligned}
$$

Setting the first law of motion equal to zero and solving for $q$ gives the locus of pairs $(Y, q)$ that constitute temporary goods market equilibrium. As $q$ impacts positively on aggregate demand (while in the familiar textbook story the interest rate impacts negatively on $Y^{d}$ ), this IS-curve is upward-sloping (rather than downward-sloping, when $Y^{d}$ depends on $i$ ). In addition, if $q$ is held constant, then all non-equilibrium points are attracted by the IS-curve. Analogously, by setting the second law of motion equal to zero the locus of pairs $(Y, q)$ can be obtained for which the stock market is in temporary equilibrium - in conjunction with money market equilibrium. Although $i(Y)$ was said to determined by the central bank's monetary policy, it will be convenient from now on to call the locus of pairs $(Y, q)$ giving rise to $(4.8)=0$ an LM-curve. ${ }^{2}$

The $\dot{q}=0$-curve, however, is unstable: Suppose share prices and, thus, Tobin's $q$ are so high that $i(Y) q-r(Y)>0$, which says that the short-term interest rate $i$ exceeds the direct returns $r p K$ from holding equities when these are related to the value of shares (their rate of return being given by $\left.r, p K / p_{e} E=r / q\right)$. In this situation share prices are driven up even higher, according to $\dot{q}>0$ in equation (19). Likewise, adjustments take place in the other direction if the right-hand side of equation (19) is negative.

The equality $\dot{q}=0$ can be explicitly solved for $q$, so that the LM-equilibrium value of $q=q_{L M}$ with respect to output $Y$ is given by the function

$$
q_{L M}(Y)=r(Y) / i(Y) .
$$

2 It should be stressed that this LM-curve represents money market and stock market equilibrium and is thus more complex than the usual LM-curve of the textbook literature (and in particular not always positively sloped as in the case of the simple money market LM-curve, see below). 
Depending on the relative (positive) slopes of $r($.$) and i(),. q_{L M}$ may rise or fall as $Y$ increases. Blanchard calls the first case, $q_{L M}^{\prime}>0$, the Good News Case, and the second, $q_{L M}^{\prime}<0$, the Bad News Case.

Figure I: Blanchard's Bad News Case with a uniquely determined steady state and Blanchard's Good News Case - with two or no steady states
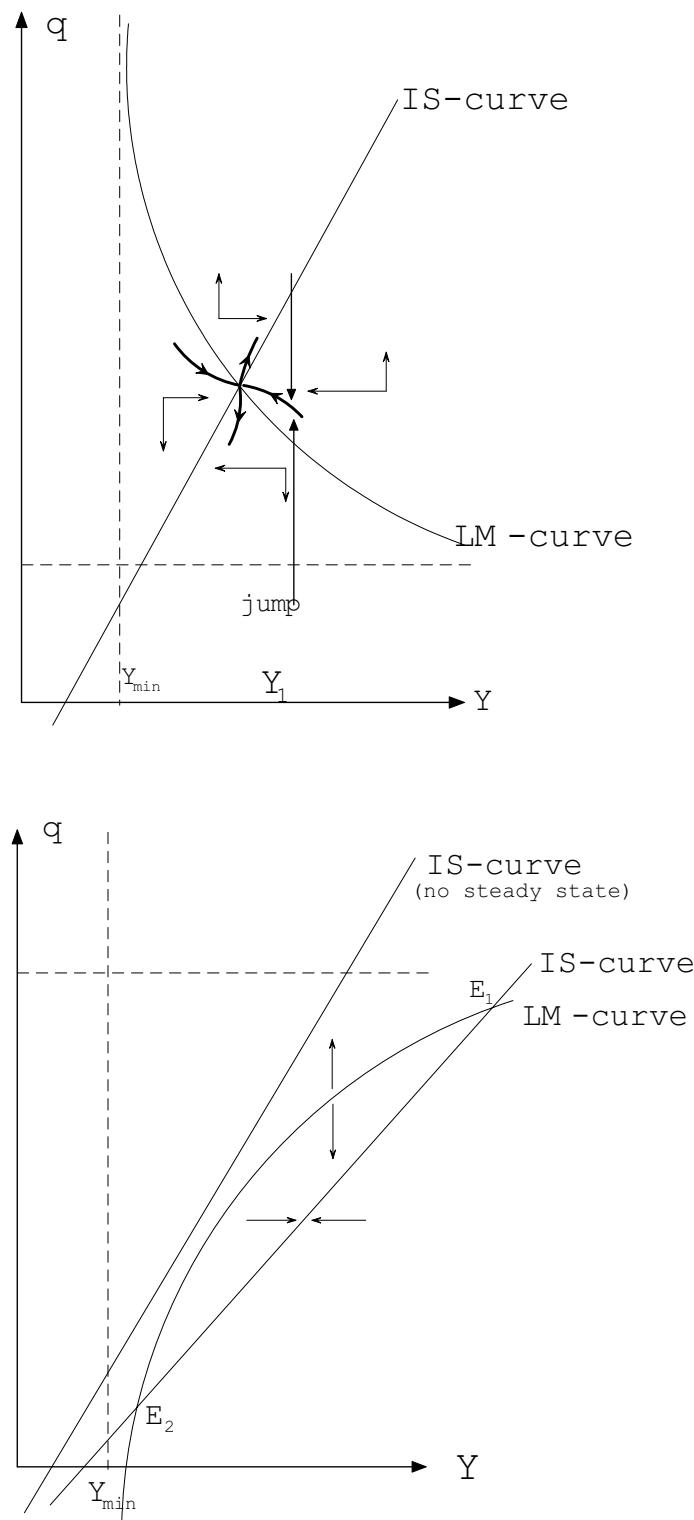


\section{Proposition}

In the Bad News Case, $q_{L M}^{\prime}<0$, the (unique) steady state of the model is a saddle point. In the Good News Case $q_{L M}^{\prime}>0$, with two equilibria. The equilibrium at which the LM-curve cuts the IS-curve from above (point $E_{1}$ in Figure $I$ is a saddle point. The other equilibrium, where the LM-curve cuts the IS-curve from below (point $E_{2}$ in Figure I), is locally asymptotically stable if $i^{o}=i\left(Y^{o}\right)<\beta_{y}\left(1-a_{y}\right)$, while it is repelling if this inequality is reversed.

Proof: The Jacobian matrix evaluated at an equilibrium point $\left(Y^{o}, q^{o}\right)$ with associated shortterm interest rate $i^{o}=i\left(Y^{o}\right)$ is given by

$$
J=\left[\begin{array}{ll}
J_{11} & J_{12} \\
J_{21} & J_{22}
\end{array}\right]=\left[\begin{array}{cc}
-\beta_{y}\left(1-a_{y}\right) & \beta_{y} a_{q} \\
i_{y} q^{o}-r_{y} & i^{o}
\end{array}\right]=\left[\begin{array}{cc}
- & + \\
? & +
\end{array}\right] .
$$

The slopes of the IS- and LM-curves are obtained from the Implicit Function Theorem (or more informally from the equations $J_{11} d Y+J_{12} d q=0$ for the IS-schedule, $J_{21} d Y+J_{22} d q=0$ for the LM-schedule). This gives

$$
\begin{aligned}
& \text { Slope IS }=-J_{11} / J_{12}=\left(1-a_{y}\right) / a_{q} \\
& \text { Slope LM }=-J_{21} / J_{22}=-\left(a_{m} q^{o}-a_{r}\right) / i^{o} .
\end{aligned}
$$

As LM is downward-sloping in the bad news case, we have $J_{21}>0$ and thus $\operatorname{det} J<0$, so that the equilibrium is a saddle point. In the good news case, Slope IS > Slope LM at an equilibrium is equivalent to $-\operatorname{det} J=-J_{11} J_{12}+J_{21} J_{22}>0$, that is, this point is a saddle too. If Slope IS < Slope LM, det $J>0$ results, so that here local stability depends on the trace. $i^{\circ}<\beta_{y}\left(1-a_{y}\right)$ rewrites the second condition, trace $J<0$, which is now necessary and sufficient for both eigen-values to have negative real parts. ${ }^{3}$

Figure 2 sketches the global dynamics that may arise in the presence of two equilibria in the good news case, assuming local stability for the lower equilibrium, which seems only natural in light of the condition stated in the proposition.

Note here that the difference in the slopes of the IS- and the LM-curve is given by:

$$
I S^{\prime}-L M^{\prime}=-\frac{J_{11}}{J_{12}}+\frac{J_{21}}{J_{22}}=-\frac{\operatorname{det} J}{J_{12} J_{22}} \text {. }
$$


Figure 2: Dynamics in the good news case: Saddle point and stable node or focus ( $E_{1}$ is the Good New Case (GNC) and $E_{2}$ the Bad Stable Equilibrium (BSE))

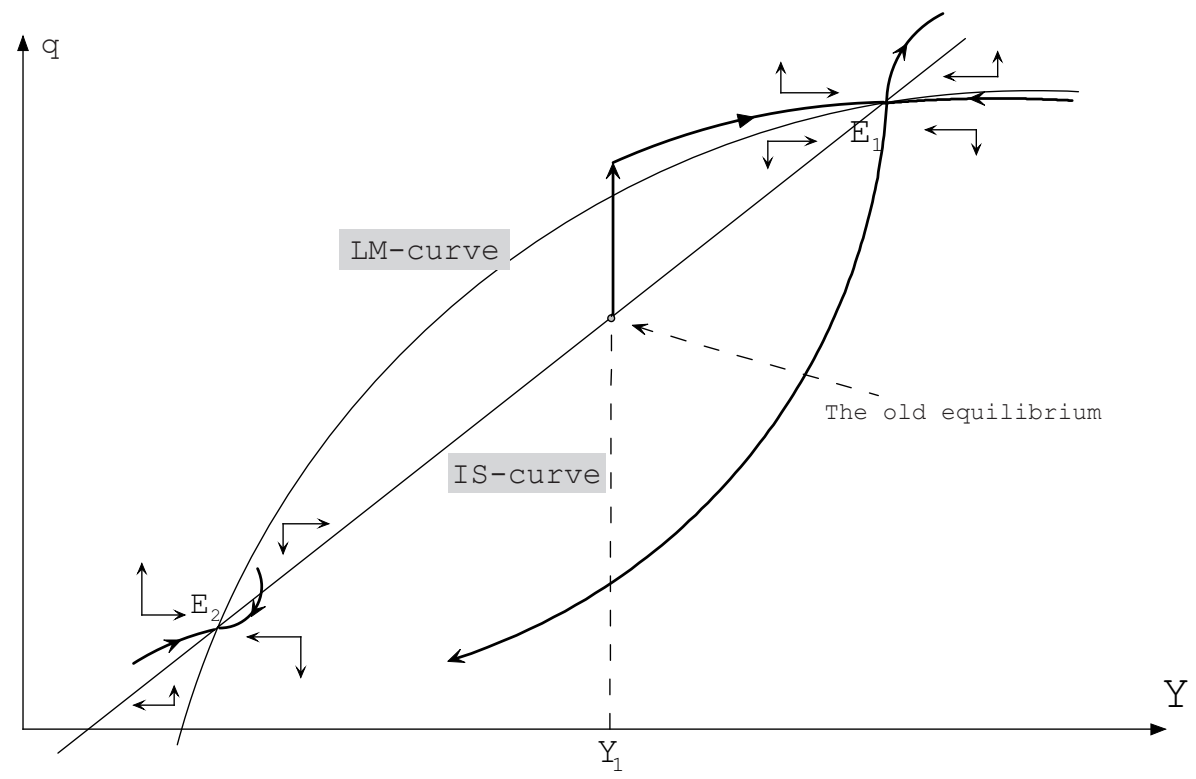

In the good news case, Blanchard (I98I: 134) himself concentrates on the upper equilibrium $E_{1}$ with its saddle point dynamics. ${ }^{4}$ The lower equilibrium $E_{2}$ is ruled out because of its undesirable comparative statics. It is indeed readily verified that an increase in the money supply or in the coefficient $c_{y}$ in (1), representing expansionary fiscal policy, both have a counterintuitive depressing effect on the lower equilibrium output. ${ }^{5}$

Blanchard (I98I) discusses a variety of applications of the jump variable technique to issues of unanticipated or anticipated fiscal or monetary policy. ${ }^{6}$ In all analysed cases, the

4 In the good news case, the long-run equilibrium $\dot{Y}=\dot{q}=0$ is unique if the LM-curve is tangent to the IS-curve. Of course, this can only happen by a fluke. It may furthermore be remarked that the problem of two or no equilibria is due to the special circumstances in the present, simplified framework. As a matter of fact, the phenomena seem to be rather spurious. Existence and uniqueness are easily reestablished if the model is put in a broader (growth) perspective where, in particular, $K$ and $E$ vary over time. In this case, the motions of $q$ are no longer so tightly linked to $p_{e}$, and the equilibrium value of $q$ is formally derived in another part of the model.

5 Blanchard adds that the dynamic properties are undesirable as well. This statement is to be understood from the point of view of the jump variable technique as the obligatory method to apply. Under asymptotic stability, this technique would no longer be determinate since the jump variable may jump anywhere and the system would still converge. Incidentally, Blanchard (1981: 135) calls the jump variable technique "a standard if not entirely convincing practice."

6 See Chiarella, Flaschel, Franke and Semmler (2009), for a discussion of these cases and the attractive and the bizarre features of this jump variable technique of the rational expectations approach, 
relevant price variable, Tobin's $q$, always jumps back instantaneously onto the stable manifold of the saddle point dynamics if the economy is perturbed from it (or the equilibrium itself) by an exogenous shock. The jump then takes the system to such a point in the phase space, that $(Y, q)$ reaches from there the stable branch of the new dynamics exactly at that point in time when the policy action (the shock) is implemented. The interesting point to be made is that the jump of $q$ in the anticipated policy case is smaller in amount than in the unanticipated case, since part of the adjustments are already smoothly carried out by the old dynamics. Such a solution of the treatment of anticipated events is, from a technical perspective, appealing. Nevertheless, the general question for the economic meaning of the jump variable procedure remains open.

While this kind of reasoning is not entirely convincing, as Blanchard acknowledges, more recent modelling is explicitly based on an intertemporal optimisation problem over an infinite horizon. ${ }^{7}$ If this problem is well-defined, the dynamic optimality conditions, again, give rise to saddle point dynamics around the steady state. In this context, jumping onto the stable manifold is a constituent part of the solution procedure. The modern treatment is consistent, but what is still problematic are the underlying assumptions. On the one hand, the hypothesis of perfect foresight, if taken literally, would demand a number of rather heroic capabilities from agents. On the other hand, the method rests on the representative agent, which is perhaps even more questionable in modelling decentralised economic systems than the requirement of perfect foresight (Kirman 1992).

The achievement of Blanchard's contribution lies in enriching the IS-LM framework by markets for equities and long-term bonds. Regarding the stock market, Figure 3 indicates an elementary destabilising mechanism, which explicitly involves expectations. An increasing expected rate of return on equities raises demand and so drives up the price on this market. As the rise in capital gains is expected to continue, expected capital gains are rising, too, and so does the expected rate of return. On the whole, a positive feedback loop is obtained. In Blanchard's treatment of the financial sector, this mechanism is not so clearly visible because he assumes perfect substitutability of all non-money assets as well as myopic perfect foresight of capital gains. As a consequence, there is no distinction between actual and expected capital gains, and the reaction mechanism disappears after some manipulation in a mathematical formula. Our approach, by contrast, relaxes Blanchard's assumptions in such a way that the individual links of the feedback chain are recovered in Figure 3.

whose construction for example then shows that an announced future change of the interest rate $i$ leads to an upward jump in the value of Tobin's $q$ and thereafter further increases now in stock prices as well as output such that the stable manifold of the new (after shock) dynamics is reached exactly when the change in the interest rate actually takes place.

7 In the early contributions to the literature, as in the seminal article by Sargent and Wallace (1973), the agents are said to consider the situation from the global perspective and, being endowed with perfect foresight, decide that all trajectories up to the stable arms of the saddle point dynamics have undesirable properties. For example, some variables would turn negative in the long-run or policy intervention may be feared. This induces "the agents" to choose the convergent path, on which the economy lands by a sudden change in the variable(s) that is (are) not predetermined in the short-run. 
The situation on the market for long-term bonds is analogous and thus need not be discussed any further. Also, following Blanchard, real investment and consumption are supposed to be independent of the long-term rate of interest, so that this rate does not feed back on the real sector.

To sum up, both in Blanchard's model and in our version a basically unstable financial sector is coupled with a stable real sector. As mentioned above, in Blanchard's approach to this interplay gives rise to saddle point dynamics, and the instability problem is solved by applying the jump variable technique. In our framework, local stability of the equilibrium becomes possible, though instability may still be considered the normal case. So we have to turn to the global dynamics. We propose an economically meaningful concept of nonlinear price reactions on the stock market that prevent the system from exploding. It is intuitively clear that when the stabilising forces are ruling in the outer regions of the state space, while in the vicinity of the equilibrium the destabilising forces remain dominant, the trajectories will undergo persistent and bounded fluctuations. Whereas their main source lies in the financial sector, a complete discussion has to take the interplay of the real and financial sector into account.

Figure 3: Centrifugal stock market dynamics

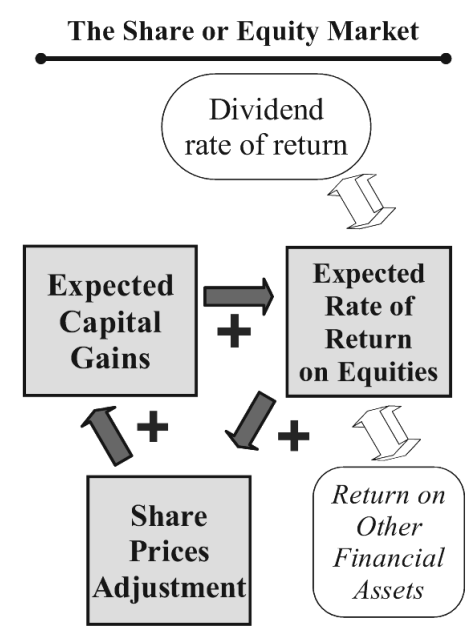




\subsection{Stability analysis for the baseline portfolio case}

Let us now go back to our portfolio model and consider first the case where $\beta_{e} \alpha_{e}$ is large enough to imply: ${ }^{8}$

$$
J=\left(\begin{array}{ccc}
J_{11} & J_{12} & J_{13} \\
J_{21} & J_{22} & J_{23} \\
J_{31} & J_{32} & J_{33}^{+}-J_{33}^{-}
\end{array}\right)=\left(\begin{array}{ccc}
- & + & 0 \\
+ & - & + \\
+ & - & +
\end{array}\right)
$$

as a situation in which case we get for the determinant

$$
|J|=\left|\begin{array}{ccc}
- & + & 0 \\
+ & - & + \\
0 & 0 & +
\end{array}\right|=\left|\begin{array}{ll}
- & + \\
+ & -
\end{array}\right|=\beta_{y}\left|\begin{array}{cc}
a_{y}-1 & a_{q} \\
r_{y} & -r_{o}
\end{array}\right|
$$

as far as signs are concerned. This expression has a negative sign if the marginal propensity to spend is sufficiently close to I. However, from the viewpoint of dimensions, one has to note that the parameter $a_{q}$ implicitly contains a level of magnitude which we, for the time being, expressed by $Y_{o}($ or $K)$. A reasonable size for $\tilde{a}_{q}=a_{q} / Y_{o}$ could then be 0.05 which implies for

$$
\left|\begin{array}{cc}
a_{y}-1 & a_{q} \\
r_{y} & -r_{o}
\end{array}\right|=r_{o}\left(1-a_{y}-\tilde{a}_{q}\right)
$$

which may be assumed as positive if savings propensities are sufficiently strong. We then have instability not only if $\beta_{\pi}$ is chosen sufficiently large (a positive trace), but also through the sign of the determinant which is the positive. And $J_{1}+J_{2}+J_{3}$ may be negative due to the other minors of order two. From the economic perspective, the expression $-J_{21} J_{12}<0$ represents an unstable real-financial market interaction, and $J_{11} J_{33}<0, J_{22} J_{33}<0$ are just the accelerating expectations mechanism in the financial markets.

We compare the neoclassical case of the preceding section with the introduction of Keynes' General Theory, where he states:

"I have called this book the General Theory of Employment, Interest and Money, placing the emphasis on the prefix general. The object of such a title is to contrast the character of my arguments and conclusions with those of the classical theory of the subject, upon which I was brought up and which dominates the economic thought, both practical and theoretical, of the governing and academic classes of this generation, as it has for a hundred years past. I shall argue that the postulates of the classical theory are applicable to a special case only and not to the general case, the situation which it assumes being a limiting point of the possible positions of equilibrium. Moreover, the characteristics of the special case assumed by the classical

8 Note that we have separated symbolically the positive effect in $J_{33}$ from the negative one in the matrix $J$. 
theory happen not to be those of the economic society which we actually live, with the result that its teaching is misleading and disastrous if we attempt to apply it to the facts of experience." (Keynes 1936: 3 )

The stability conditions (for the determinant) in the case of the jump variable technique (which always place the economy on the stable arm of a saddle-point scenario) are-as shown abovejust opposite in sign to the determinant of the portfolio adjustment case and thus represent polar - indeed even incompatible - cases of each other. This provides a contemporaneous illustration for the above statement of Keynes, implying here that the stability illusion of the rational expectations school is one of the reasons why the macroeconomics profession has not been prepared to consider the occurrence of deep financial and real crises as they have just occurred on a worldwide level.

Whereas rational expectations scenarios such as the one of the preceding section are by assumption stable ones, we now use our portfolio approach to Keynesian macrodynamics to discuss policy measures that can stabilise this economy also in the case of a repelling stationary state, a topic that is simply absent in the case of its neoclassical limit case.

\section{Fiscal policy, financial and real stability}

In view of the feedback structure of the portfolio-multiplier dynamics, we first impose a capital gains tax $\tau_{e}$ on the system and get as law of motion of capital gain expectations in such a situation

$$
\dot{\pi}_{e}^{e}=\beta_{\pi}\left[\left(1-\tau_{e}\right) \hat{q}-\pi_{e}^{e}\right]
$$

as reduced adjustment process towards the actual capital gains of asset holders. This gives rise to a new dynamical system:

$$
\begin{aligned}
\dot{Y} & =\beta_{y}\left[\left(a_{y} Y+a_{q}\left(q-q_{o}\right)+\bar{G}-Y\right)\right], \\
\hat{q} & =\beta_{e} \alpha_{e}\left[f_{e}\left(r(Y) / q+\pi_{e}^{e}\right)\left(1+\frac{M_{2}}{p_{e} \bar{E}}\right)-1\right], \\
\dot{\pi}_{e}^{e} & =\beta_{\pi}\left[\left(1-\tau_{e}\right) \hat{q}-\pi_{e}^{e}\right],
\end{aligned}
$$

with the new Jacobian:

$$
J=\left(\begin{array}{ccc}
J_{11} & J_{12} & J_{13} \\
J_{21} & J_{22} & J_{23} \\
\left(1-\tau_{e}\right) J_{31} & \left(1-\tau_{e}\right) J_{32} & \left(1-\tau_{e}\right) J_{33}^{+}-J_{33}^{-}
\end{array}\right)=\left(\begin{array}{ccc}
- & 0 \\
+ & - & + \\
+ & - & -
\end{array}\right)
$$

if there holds $\left(1-\tau_{e}\right) \beta_{e} \alpha_{e} f_{e}^{\prime}(\cdot)\left(1+\frac{M_{2}}{p_{e} \bar{E}}\right)-1<0$, i.e., 


$$
1-\frac{1}{\beta_{e} \alpha_{e} f_{e}^{\prime}(\cdot)\left(1+\frac{M_{2}}{p_{e} \bar{E}}\right)}<\tau_{e}
$$

The system is then a stable one if there holds in addition to what we have already assumed above: ${ }^{9}$

$$
\left|\begin{array}{cc}
a_{y}-1 & a_{q} \\
r_{y} & -r_{o}
\end{array}\right|>0
$$

since the trace is negative, the minors of order two are then all positive, the determinant is negative, and finally the $a_{1} a_{2}-a_{3}$ Routh-Hurwitz term is positive. ${ }^{\text {Io }}$ This holds if the following increasingly stronger conditions hold true:

$$
\begin{aligned}
& \left(J_{1}+J_{2}+J_{3}\right)\left(-J_{11}-J_{22}-J_{33}\right)+J_{3} J_{33}^{-}>0, \\
& J_{3}\left(-J_{11}-J_{22}-J_{33}\right)>-J_{3} J_{33}^{-}, \\
& -J_{11}-J_{22}-J_{33}>-J_{33}^{-} \\
& -J_{11}-J_{22}>-J_{33}^{+}, \\
& \beta_{y} a_{y}+\beta_{e} \alpha_{e} f_{e}^{\prime}\left(r_{o}\right)\left(1+\frac{M_{2}}{p_{e} \bar{E}}\right)>\left(1-\tau_{e}\right) \beta_{\pi} .
\end{aligned}
$$

We thus get that all adjustment speeds (and more) are involved in the establishment of the local asymptotic stability of the steady state.

Again, the adjustment speed of capital gain expectations is the crucial destabilising force which can be counteracted by an increase of the adjustment speeds in the goods as well as in the stock market. In addition, taxing capital gains removes the expectations accelerating mechanism from the dynamics, but needs again of course the additional condition on the feedback loop between the share price dynamics and the investment behaviour of firms in order to establish convergence to the steady state. A Taylor rule is of no importance here since it only affects the cash management of the asset holders, but not the real financial market interaction.

In order to also tame this last positive feedback loop further, we may assume in addition that the government runs a countercyclical fiscal policy: $G(Y), G^{\prime}<0$ which makes the term $J_{11}$ more negative. One may also attempt to increase the savings propensity of households which has the same effect. Finally, the addition of fundamentalists' expectations of stock appreciations can also lead to further stabilising elements in the considered dynamics.

9 This is again just the opposite sign of the neoclassical rational expectations case.

Io This also needs a multiplier parameter $\beta_{y}$ that is sufficiently high, a condition that is fulfilled when the economy is close to an IS-equilibrium configuration. 
The overall result is that monetary policy is of no use in the present type of a real financial market interaction, while tax policies can bring stability to the system if the interaction between output and share prices is a stable one when capital gain expectations are given. We could however have an effect of the rate of interest on the demand function $f_{e}$, not as a substitution effect, but as a climate effect if the lowering the interest rate would be interpreted by the stock market participants as an improvement of the state of the economy and thus as improvement of the future profitability of firms.

\section{Monetary policy, stock markets and the public state of confidence}

We thus assume now that stock holders interpret high interest rates $i$, obtained from a Taylor rule of type $i(Y)$, as bad and vice versa, and as representing the official state of confidence of the monetary authority. The interest rate therefore then enters the equity demand function in a negative way

$$
\hat{q}=\beta_{e} \alpha_{e}\left[f_{e}\left(i(Y), r(Y) / q+\pi_{e}^{e}\right)\left(1+\frac{M_{2}}{p_{e} \bar{E}}\right)-1\right],
$$

which however had nothing to do with imperfect substitutability between the structure of $M_{2}$ and the equity market. In this way the so far totally ineffective monetary policy can become effective, though open market operations do not move anything outside the money supply $M_{2}$.

The above adds a term of type $f_{e, 1} i^{\prime}(Y)<0$ to the entry $J_{21}$ of the Jacobian $J$ and is thus stabilising in a fairly direct way, if not counteracted by the capital gain expectations of asset holders. It makes the following determinant more negative,

$$
\left|\begin{array}{cc}
a_{y}-1 & a_{q} \\
r_{y} & -r_{o}
\end{array}\right|>0
$$

and contributes thereby to the overall stability of the considered dynamic interaction of the goods with the asset markets.

Keynes (1936) already in addition proposed in the General Theory:

"Where, however, (as in the United States, 1933-1934) open-market operations have been limited to the purchase of very short-dated securities, the effect may, of course, be mainly confined to the very short-term rate of interest and have but little reaction on the much more important long-term rates of interest." (Keynes 1936: 197)

Following this quotation, long-term bonds should now also be included. This topic is treated in Charpe, Flaschel, Hartmann and Proaño (20I0), but can be analogised here by assuming instead that the central bank is even prepared to trade in stocks in a countercyclical way by way of open market operations. This case in investigated in Asada et al. (2010) in the context 
of a KMG Tobin model and gives rise to stability results that also apply to the present case of only a dynamic multiplier/portfolio choice interaction.

\section{Conclusions}

We have reconsidered in this paper the seminal model of real-financial interaction put forward by Blanchard (198I), which extends the IS analysis by an output adjustment rule of the dynamic multiplier type, and, more importantly, the textbook LM analysis by stock market dynamics under the assumption of perfect substitutability and myopic perfect foresight. As widely known, this system is typically characterised by saddle point dynamics and the use of the jump-variable technique in order to guarantee its stability (by assumption).

It is however very questionable - in particular against the background of our own model - whether such ideal assumptions and stability constructions can be relevant for the study of actual economies, in particular of the present financial market turbulences. The interest rate on short-term bonds is here influencing the stock market as a direct portfolio alternative to equity holding (not as a business cycle climate expression). This would not only restrict capital gains quantitatively to a very narrow range, but also imply that simple cash management procedure can become of decisive importance for the working of the stock market (and the market for bonds when added to this situation as in Blanchard (I98I). Moreover, the assumption of myopic perfect foresight is here turned into a global behavioural rule, since asset holders now have to overlook the whole phase space in order to see to which bubble they should jump to land on the new stable arm in time (when anticipated shocks are considered).

We believe that the model we have been proposing in the body of this paper is descriptively much more correct than such an ideal situation and its analytical treatment by way of the jump variable technique is a redundant issue. Moreover, though we have considered the latter situation by setting certain parameters equal to infinity, the limit case is not at all related to what is happening for very large, but still finite adjustment speeds. There is not only the fact that the resulting ideal equations (in place of the laws of motion of the situation with imperfections) represent a discontinuity in phase diagram properties, where moreover the solution method is then switched from only predetermined variables to one where there is now one non-predetermined jump variable in the financial market subsystem. There is thus no bridge possible between the neoclassical limit case and our model of imperfect substitutes and imperfect information, concerning the investigation of appropriate policy measures in order to stabilise an unstable economy. 


\section{Notation}

Steady state are indicated by a subscript $` \propto$, exogenous variables by an upper bar and demand by a superscript $>d$. A dot over a variable $x=x(t)$ denotes the time derivative, a caret its growth rate; $\dot{x}=d x / d t, \hat{x}=\dot{x} / x$.

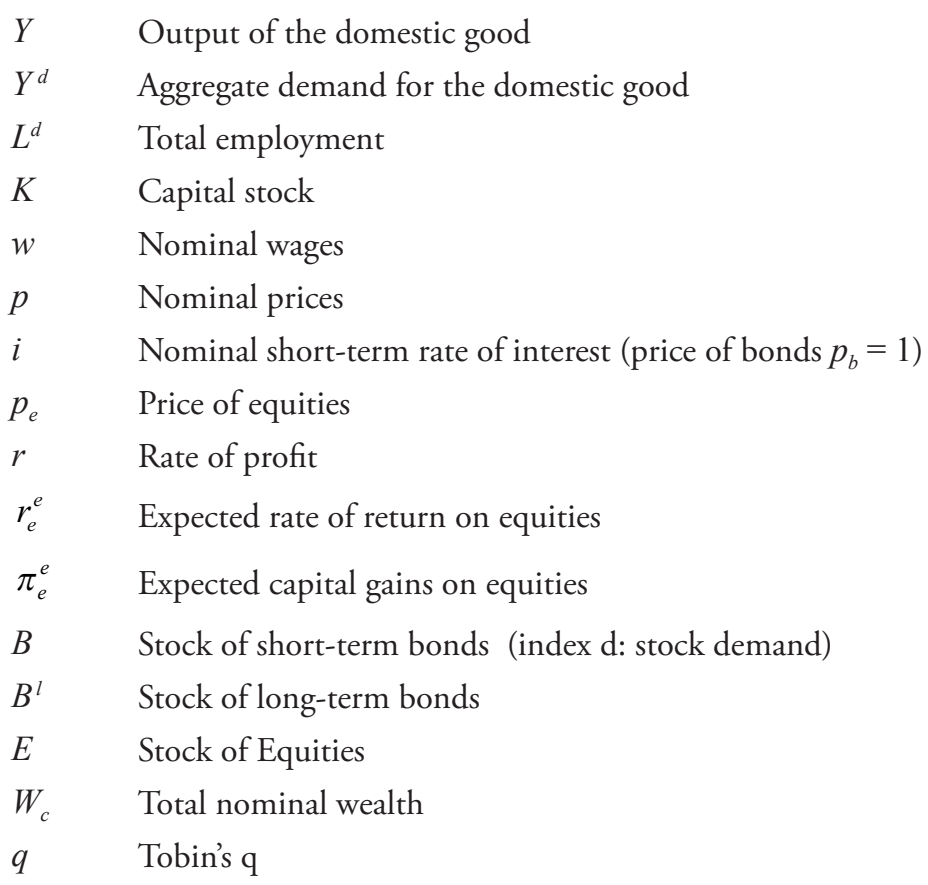

\section{References}

Asada, T., Flaschel, P., Mouakil, T., Proaño, C.R. (2010): Asset Markets, Portfolio Choice and Macroeconomic Activity. A Keynesian Perspective, Houndmills/Basingstoke: Palgrave Macmillan.

Blanchard, O. (1981): Output, the stock market, and interest rates, in: American Economic Review, $7 \mathrm{I}(\mathrm{I}), \mathrm{I} 32-43$.

Charpe, M., Flaschel, P., Hartmann, F, Proańo, C.R. (20ı): Stabilizing an unstable economy: Fiscal and monetary policy, stocks, and the term structure of interest rates, CEMM Bielefeld Working paper.

Chiarella, C., Flaschel, P., Franke, R., Semmler, W. (2009): Financial Markets and the Macroeconomy. A Keynesian Perspective, London: Routledge.

Keynes, J.M. (1936): The General Theory of Employment, Interest and Money, New York: Macmillan. Kirman, A.P. (1992): Whom or what does the representative individual represent?, in: Journal of Economic Perspectives, 6, $\mathrm{I} 7-\mathrm{I} 36$. 
Sargent, T. (1987): Macroeconomic Theory, New York: Academic Press, $2^{\text {nd }}$ edn.

Sargent, T., Wallace, N. (1973): The stability of models of money and growth with perfect foresight, in: Econometrica, 4I, IO43-48.

Tobin, J. (1982): Money and the macroeconomic process, in: Journal of Money, Credit and Banking, I4, I7I-204.

Turnovsky, S. (I995): Methods of Macroeconomic Dynamics, Cambridge, MA: The MIT Press. 